PROCEEDINGS OF THE

AMERICAN MATHEMATICAL SOCIETY

Volume 126, Number 11, November 1998, Pages 3271-3278

S 0002-9939(98)04572-9

\title{
SPECTRAL CONDITIONS GUARANTEEING A NONTRIVIAL SOLUTION OF THE ABSTRACT CAUCHY PROBLEM
}

\author{
R. DELAUBENFELS AND S. WANG
}

(Communicated by Palle E. T. Jorgensen)

\begin{abstract}
We characterize subsets, $\Omega$, of the complex plane, with the following property: If $A$ has spectrum contained in $\Omega$, with polynomially bounded resolvent outside $\Omega$, then the abstract Cauchy problem corresponding to $A$ has a nontrivial solution.
\end{abstract}

\section{INTRODUCTION AND PRELIMINARIES}

Many major physical problems, including initial-value problems, mixed initialboundary-value problems, and other integrodifferential equations, may be modelled as an abstract Cauchy problem:

$$
\frac{d}{d t} u(t)=A(u(t))(t \geq 0), u(0)=x .
$$

See, for example, [5], [6] or [7]. This reduces the original problem to a problem in operator theory: what conditions on the operator $A$ will guarantee that (ACP) has a unique solution?

One of the most natural ways to study a linear operator is by looking at its spectrum. The goal is to think of $A$ as a complex number, or a set of complex numbers.

In this paper, we will discuss what conditions on the spectrum of $A$ will guarantee a nontrivial solution of (ACP). We will not discuss uniqueness. Relatively weak conditions on $A$ will guarantee uniqueness; see, for example, [6, Theorem 4.1.2].

For unbounded operators, it is not sufficient to specify the location of the spectrum; one must also control the rate of growth of the norm of the resolvent, $\left\|(z-A)^{-1}\right\|$, as $|z| \rightarrow \infty$. We will assume polynomial growth of the resolvent, outside a given set.

It is convenient to introduce terminology and hypotheses before proceeding.

Terminology and Hypotheses 1.0. All operators are linear, on a Banach space $X$. We will write $\mathcal{D}(A)$ for the domain of the operator $A, \operatorname{Im}(A)$ for the image of $A, \sigma(A)$ for the spectrum, and $\rho(A)$ for the resolvent. $B(X)$ will be the space of bounded operators from $X$ into itself.

Throughout this paper, $\Omega$ and $O$ are open subsets of the complex plane, whose complement contains a half-line and whose boundaries, $\partial \Omega$ and $\partial O$, are positively

Received by the editors June 12, 1996 and, in revised form, March 20, 1997.

1991 Mathematics Subject Classification. Primary 47D03, 34G10, 47D06, 47A60.

(C)1998 American Mathematical Society 
oriented countable systems of piecewise-smooth, mutually nonintersecting (possibly unbounded) arcs, and $\alpha$ is a real number greater than or equal to -1 .

By a solution of $(\mathrm{ACP})$ we mean a strong solution; that is, $u \in C^{1}([0, \infty), X) \cap$ $C([0, \infty),[\mathcal{D}(A)])$ and satisfies $(\mathrm{ACP})$, where $\|x\|_{[\mathcal{D}(A)]} \equiv\|x\|+\|A x\|$.

Definition 1.1 ([2, Definition 20.1]). The operator $B$ is of $\alpha$-type $\Omega$ if $\sigma(B) \subseteq \Omega$ and there exists a constant $M$ so that

$$
\left\|(\lambda-B)^{-1}\right\| \leq M(1+|\lambda|)^{\alpha}, \forall \lambda \notin \Omega .
$$

Note that an operator of $\alpha$-type $\Omega$ may or may not be densely defined.

For any nonnegative integer $m$, let $H_{m}^{\infty}(\Omega)$ be the set of all functions $f$ from $\Omega$ into the complex plane such that

$$
z \mapsto(\lambda-z)^{m} f(z) \in H^{\infty}(\Omega),
$$

for any $\lambda \notin \bar{\Omega}$.

Throughout this paper, we will use the following generalized Riesz-Dunford functional calculus.

Definition 1.2. If $A$ is of $\alpha$-type $\Omega, m \equiv[\alpha]+2$ and $h \in H_{m}^{\infty}(\Omega)$, define $h(A) \in$ $B(X)$ by

$$
h(A) \equiv \int_{\partial O} h(w)(w-A)^{-1} \frac{d w}{2 \pi i},
$$

where $O$ is chosen so that $\bar{O} \subseteq \Omega$ and $A$ is of $\alpha$-type $O$ (see [2, Lemma 22.4]).

Note that $h \in H_{m}^{\infty}(\Omega)$ need not be analytic at $\infty$.

See [4, Example 4.1] and [2, Definition 22.6] for equivalent definitions of $h(A)$ that make sense with the growth conditions on $h$ removed.

Definition 1.3. The strongly continuous family $\{W(t)\}_{t \geq 0} \subseteq B(X)$ is a $C$ regularized semigroup for $A$ if

(1) $W(0)=C$;

(2) $W(t) W(s)=C W(t+s)$, for all $s, t \geq 0$;

(3) $W(t) A \subseteq A W(t)$, for all $t \geq 0$; and

(4) for any $x \in X, t \geq 0, \int_{0}^{t} W(s) x d s \in \mathcal{D}(A)$, with

$$
A\left(\int_{0}^{t} W(s) x d s\right)=W(t) x-C x .
$$

$C$-regularized semigroups may be used to deal with ill-posed abstract Cauchy problems in the same way that strongly continuous semigroups deal with well-posed problems. In particular, when there exists a $C$-regularized semigroup $\{W(t)\}_{t \geq 0}$ for $A$ and $A$ is closed, we are guaranteed solutions of (ACP) for all $x$ in $C(\mathcal{D}(A))$,

$$
u(t) \equiv W(t) y(C y=x)
$$

and well-posedness on a Fréchet space (a Banach space, if the regularized semigroup is exponentially bounded) continuously embedded between $\operatorname{Im}(C)$ and $X$. See [3], and, for $C$ injective, [2].

We will characterize those subsets of the complex plane with the following property: When $A$ is of $\alpha$-type $\Omega$, then (ACP) has a nontrivial solution. We shall see that it is sufficient that there exist nontrivial $h$ such that

$$
z \mapsto e^{t z} h(z) \in H^{\infty}(\Omega)
$$


for all nonnegative $t$ (Corollary 2.4). A slight weakening produces a necessary and sufficient condition (Theorem 2.7). It then follows automatically that there exists a nontrivial operator $g(A)$ such that

$$
W(t) \equiv\left(z \mapsto e^{t z} g(z)\right)(A)(t \geq 0)
$$

defines a $g(A)$-regularized semigroup for $A$ (Theorems 2.3 and 2.7). Thus, one nontrivial solution leads to the existence of solutions for all initial data in the image of $g(A)$, represented by a regularized semigroup constructed explicitly with a functional calculus

$$
u(t)=W(t) y=\int_{\partial O} e^{t w} g(w)(w-A)^{-1} y \frac{d w}{2 \pi i},
$$

where $W(0) y=g(A) y=x$.

We mentioned earlier that the abstract Cauchy problem reduces a physical problem to a problem in operator theory; Theorem 2.7 further reduces it to a problem in complex analysis: On which subsets of the complex plane do there exist nontrivial holomorphic functions that decay more rapidly than $z \mapsto e^{t z}$, for all nonnegative $t$ ?

Intuitively, one thinks of a solution of (ACP) as $u(t)=e^{t A} x$. Spectral intuition suggests that we think of $A$ as multiplication by $z$ on $\sigma(A)$; a solution of (ACP) is then thought of, very informally, as

$$
t \mapsto\left(z \mapsto e^{t z} g(z)\right) \quad(z \in \sigma(A)),
$$

for some function $g$. Our results are consistent with this intuition.

One way to regard these results is as indicating the omnipresence of regularized semigroups. If spectral conditions guarantee a nontrivial solution of (ACP), then the solution is accessible via a regularized semigroup for $A$.

\section{MAIN RESUlTS}

When considering operators of type $\Omega$, we will focus on sets $\Omega$ that have no extraneous connected components; that is, all connected components have nontrivial intersection with $\sigma(A)$ (see Example 2.9(e)).

Definition 2.1. We will say that $\Omega$ is an $\alpha$-spectral solution set if, whenever $A$ is of $\alpha$-type $\Omega$, and $\sigma(A) \cap \Omega_{j}$ is nontrivial when $\Omega_{j}$ is a connected component of $\Omega$, then $(\mathrm{ACP})$ has a nontrivial solution.

Definition 2.2 ([2, Definition 23.2]). We define $E_{\Omega}$ to be the set of all functions $h$ from $\Omega$ into the complex plane such that

$$
z \mapsto e^{t z} h(z) \in H^{\infty}(\Omega)
$$

for all $t \geq 0$.

Theorem 2.3. Suppose

(1) $E_{\Omega}$ is nontrivial;

(2) $A$ is of $\alpha$-type $\Omega$; and

(3) $\sigma(A) \cap \Omega_{j}$ is nontrivial whenever $\Omega_{j}$ is a connected component of $\Omega$.

Then there exists $g \in E_{\Omega} \cap H_{m}^{\infty}(\Omega)(m \equiv[\alpha]+2)$ such that $g(A)$ is nontrivial and

$$
W(t) \equiv\left[\left(z \mapsto e^{t z} g(z)\right)(A)\right] \quad(t \geq 0)
$$

defines a $g(A)$-regularized semigroup for $A$.

Corollary 2.4. If $E_{\Omega}$ is nontrivial, then $\Omega$ is an $\alpha$-spectral solution set. 
Remark 2.5. With some additional conditions on $g \in E_{\Omega}, g(A)=W(0)$, in Theorem 2.3, will be injective, so that the solutions of (ACP) guaranteed by Corollary 2.4 will also be unique; see [4, Theorem 5.2].

To characterize $\alpha$-spectral solution sets, we need one more definition.

Definition 2.6 ([2, Definition 23.4]). If $\bar{O} \subseteq \Omega$, then $O$ is an $\alpha$-interior subset of $\Omega$ if there exists a constant $c>0$ such that

$$
d(O,\{z \notin \Omega|| z \mid \leq R\}) \geq c(1+R)^{-\alpha},
$$

for all $R>0$.

Theorem 2.7. The following are equivalent.

(a) $\Omega$ is an $\alpha$-spectral solution set.

(b) Whenever $A$ is of $\alpha$-type $\Omega$, and $\sigma(A) \cap \Omega_{j}$ is nontrivial when $\Omega_{j}$ is a connected component of $\Omega$, then there exists a nontrivial $C$ such that $A$ has a $C$-regularized semigroup.

(c) For all $\alpha$-interior subsets $O$, of $\Omega, E_{O}$ is nontrivial.

We then have

$$
u(t)=W(t) y=\left[\left(z \mapsto e^{t z} g(z)\right)(A)\right] y,
$$

where $u$ is a nontrivial solution of $(A C P),\{W(t)\}_{t \geq 0}$ is a $C$-regularized semigroup for $A, C y=g(A) y \neq 0$, and $g$ is an appropriate member of $E_{O}$.

Remarks 2.8. In [2, Definition 23.1], $\Omega$ is defined to be an $\alpha$-spectral dense solution set if, whenever $A$ is of $\alpha$-type $\Omega$ and densely defined, then (ACP) has a solution for all initial data in a dense set. [2, Theorem 23.6] is an analogue of Theorem 2.7(a) $\Longleftrightarrow$ (c): $\Omega$ is an $\alpha$-spectral dense solution set if and only if, for all $\alpha$-interior subsets $O$, of $\Omega, E_{O}$ is uniformly dense in $H^{\infty}(O)$.

In particular, an $\alpha$-spectral dense solution set is automatically an $\alpha$-spectral solution set.

We reiterate that there are no assumptions about density of domain in this paper. Our conditions on $\Omega$ are weaker than in [2, Theorem 23.6], because we are characterizing a weaker condition; all we are asking for in this paper is one nontrivial solution of (ACP). The results in [2, Chapter XXIII] contain no equivalences between $\alpha$-spectral dense solution sets and regularized semigroups.

Examples 2.9. (a). The sets in [2, Examples 23.13, 23.15, 23.16, 23.17, 23.18] are $\alpha$-spectral dense solution sets, hence $\alpha$-spectral solution sets, for any $\alpha \geq-1$.

(b). For some negative results, consider, as in [2, Example 2.7], $A \equiv 1+\frac{d}{d x}$, with maximal domain, on $X \equiv\{f \in C[0,1] \mid f(0)=0\}$. Since $(1-A)$ generates a bounded strongly continuous semigroup, $A$ is of 0-type $S_{\frac{\pi}{2}}$, and is of (-1)-type $S_{\theta}$, for $\pi>\theta>\frac{\pi}{2}$, where $S_{\theta} \equiv\left\{r e^{i \phi}|| \phi \mid<\theta, r>0\right\}$. However it is not hard to show that $(\mathrm{ACP})$ has no nontrivial solutions.

Thus $S_{\frac{\pi}{2}}$ is not a 0 -spectral solution set, and for $\pi>\theta>\frac{\pi}{2}, S_{\theta}$ is not an $\alpha$ spectral solution set, for any $\alpha \geq-1$. It is shown in [2, Example 2.7] that $S_{\frac{\pi}{2}}$ is a $(-1)$-spectral dense solution set. Thus $S_{\frac{\pi}{2}}$ is a $(-1)$-spectral solution set.

(c). Example 2.9(b) provides a novel way of showing the complex analysis fact that there exists no function $g$ such that

$$
z \mapsto e^{t z} g(z) \in H^{\infty}\left(S_{\frac{\pi}{2}}\right)
$$


for all $t \geq 0$; that is, $E_{S_{\frac{\pi}{2}}}$ is trivial. Note that, by [2, Example 23.15], $E_{S_{\theta}}$ is dense in $H^{\infty}\left(S_{\theta}\right)$, for any positive $\theta<\frac{\pi}{2}$.

(d). An example of a set $\Omega$ that, for any $\alpha \geq-1$, is an $\alpha$-spectral solution set but not an $\alpha$-spectral dense solution set, is

$$
\Omega \equiv \Omega_{1} \cup \Omega_{2},
$$

where $\Omega_{1} \equiv S_{\frac{2 \pi}{3}}, \Omega_{2} \equiv-S_{\frac{\pi}{6}}$. Since $1_{\Omega_{2}} \in E_{\Omega}, \Omega$ is an $\alpha$-spectral solution set, for any $\alpha \geq-1$. Since $\Omega_{1}$ is not an $\alpha$-spectral dense solution set, $\Omega$ is not an $\alpha$-spectral dense solution set, for any $\alpha \geq-1$.

(e). Note that, if $\Omega$ has a bounded connected component, then $E_{\Omega}$ is nontrivial. Thus an immediate corollary of Corollary 2.4 is that, if $A$ has polynomially bounded resolvent outside a set $\Omega$ that has a bounded connected component, then (ACP) has a nontrivial solution. We believe this is well-known, since, if $\Omega_{1}$ is a bounded connected component, then there exists a bounded spectral projection corresponding to $\Omega_{1}$ (see [1, Chapter 2.2]).

This observation also explains why, for meaningful results, the condition in Definition 2.1 on the connected components of $\Omega$ is necessary. Let $\Omega_{1}$ be any set, let $\Omega_{2}$ be a bounded set whose closure is disjoint from $\Omega_{1}$, and let

$$
\Omega \equiv \Omega_{1} \cup \Omega_{2} .
$$

Then if, for some $\alpha \geq-1, A$ is of $\alpha$-type $\Omega_{1}, A$ is also of $\alpha$-type $\Omega$. But $\Omega_{1}$, hence $A$, could be as bad as possible, e.g., $\Omega_{1} \equiv S_{\theta}$, for $\pi>\theta>\frac{\pi}{2}$ (see (b)), while $E_{\Omega}$ is nontrivial.

\section{Proofs OF THE MAIN RESUlts}

The following may be of some independent interest.

Proposition 3.1. If $\rho(A)$ is nonempty, and $\mathcal{A}$ is the Banach algebra generated by all resolvents of $A$ and the identity operator, then the maximal ideal space of $\mathcal{A}$ equals

$$
\left\{m_{\mu} \mid \mu \in \sigma(A) \cup\{\infty\}\right\},
$$

where, for $\mu \in \sigma(A)$,

$$
m_{\mu}\left((\omega-A)^{-1}\right) \equiv(\omega-\mu)^{-1} \forall \omega \in \rho(A),
$$

and $m_{\infty}$ is the complex homomorphism that annihilates all resolvents of $A$.

Proof. For $\omega \in \rho(A)$, let $\hat{R_{\omega}}$ be the Gelfand transform of the resolvent $R_{\omega} \equiv$ $(\omega-A)^{-1}$. For $\omega, \nu \in \rho(A)$, the resolvent formula implies that

$$
\hat{R_{\omega}}-\hat{R_{\nu}}=(\nu-\omega) \hat{R_{\omega}} \hat{R_{\nu}}
$$

For $m \neq m_{\infty}$ in the maximal ideal space of $\mathcal{A}$, there exists $\omega$ such that $\hat{R}_{\omega}(m) \neq 0$; thus we may define

$$
\mu \equiv \omega-\left[\hat{R_{\omega}}(m)\right]^{-1}
$$

By $\left.{ }^{* *}\right), \mu$ is independent of the choice of $\omega \in \rho(A)$. Since

$$
\sigma\left(R_{\omega}\right)=\left\{(\omega-\mu)^{-1} \mid \mu \in \sigma(A)\right\} \cup\{0\}
$$

([1, Lemma 2.11]), $\mu \in \sigma(A)$, so that $m=m_{\mu}$, as defined by $\left(^{*}\right)$. 
Conversely, suppose $\mu \in \sigma(A)$. Note first that, for a fixed $\omega_{0} \in \rho(A)$, if $B \equiv R_{\omega_{0}}$, then $\mathcal{A}$ is the Banach algebra generated by $B$ and the identity operator. This may be seen by noting that, if

$$
f_{\omega}(z) \equiv \frac{z}{z\left(\omega-\omega_{0}\right)+1},
$$

then, for any $\omega \in \rho(A), f_{\omega}(B)=R_{\omega}$, and by $\left({ }^{* * *}\right), f_{\omega}$ is analytic in a neighborhood of $\sigma(B)$.

Note also that $\left(^{*}\right)$ may be equivalently stated as

$$
m_{\mu}\left(f_{\omega}(B)\right) \equiv f_{\omega}\left(\frac{1}{\omega_{0}-\mu}\right) \forall \omega \in \rho(A)
$$

which, by $\left.{ }^{* * *}\right)$, extends to a complex homomorphism on $\mathcal{A}$.

Now we may characterize those $h$ for which the Gelfand transform of $h(A)$ (Definition 1.2) is trivial.

Proposition 3.2. Suppose $A$ is of $\alpha$-type $\Omega, m \equiv[\alpha]+2$ and $h \in H_{m}^{\infty}(\Omega)$. Then the following are equivalent.

(a) $\widehat{[h(A)]} \equiv 0$.

(b) $h \equiv 0$ on $\sigma(A)$.

In particular, if $h(A) \equiv 0$, then (b) follows.

Proof. For any $\mu \in \sigma(A)$,

$$
\begin{aligned}
m_{\mu}(h(A)) & =\int_{\partial O} h(w) m_{\mu}\left((w-A)^{-1}\right) \frac{d w}{2 \pi i} \\
& =\int_{\partial O} h(w)(w-\mu)^{-1} \frac{d w}{2 \pi i}=h(\mu) .
\end{aligned}
$$

The same calculation shows that $m_{\infty}(h(A))=0$.

By Proposition 3.1, the equivalence of (a) and (b) follows.

Proof of Theorem 2.3. Fix $\lambda \notin \Omega$.

First we will show that

$$
W(t) \equiv\left[\left(z \mapsto e^{t z} g(z)\right)(A)\right] \quad(t \geq 0)
$$

defines a $g(A)$-regularized semigroup for $A$, whenever

$$
k(z) \equiv(\lambda-z)^{m} g(z) \in E_{\Omega} .
$$

By considering separately $\operatorname{Re}(z) \leq 0$ and $\operatorname{Re}(z)>0$, it is not hard to see that

$$
t \mapsto\left(z \mapsto e^{t z} k(z)\right)
$$

is a locally bounded map from $[0, \infty)$ into $H^{\infty}(\Omega)$. Thus dominated convergence implies that $t \mapsto W(t)$ is a continuous map from $[0, \infty)$ into $B(X)$.

The fact that $h \mapsto h(A)$ is an algebra homomorphism ([2, Lemma 22.35(a)]) implies that $\{W(t)\}_{t \geq 0}$ satisfies (2) of Definition 1.3.

Since $\lambda \in \rho(A),(3)$ of Definition 1.3 is equivalent to

$$
W(t)(\lambda-A)^{-1}=(\lambda-A)^{-1} W(t)
$$

for all $t \geq 0$; this is clear from the construction of $W(t)$. 
To verify (4) of Definition 1.3, note that

$$
\begin{aligned}
\int_{0}^{t} W(s) e^{-\lambda s} d s & =\int_{\partial O}\left[\int_{0}^{t} e^{(w-\lambda) s} d s\right] g(w)(w-A)^{-1} \frac{d w}{2 \pi i} \\
& =\int_{\partial O}\left(e^{(w-\lambda) t}-1\right) g(w)(w-A)^{-1} \frac{d w}{2 \pi i(w-\lambda)} .
\end{aligned}
$$

By [2, Lemma 22.35(b)], $\operatorname{Im}\left(\int_{0}^{t} W(s) e^{-\lambda s} d s\right) \subseteq \mathcal{D}(A)$, with

$$
\begin{aligned}
(A-\lambda) \int_{0}^{t} W(s) e^{-\lambda s} d s & =\int_{\partial O}\left(e^{(w-\lambda) t}-1\right) g(w)(w-A)^{-1} \frac{d w}{2 \pi i} \\
& =e^{-\lambda t} W(t)-W(0), \quad \forall t \geq 0 .
\end{aligned}
$$

We translate from $(A-\lambda)$ to $A$ as follows. Applying $(A-\lambda)^{-1}$ to both sides of $(* *)$ and differentiating gives us

$$
W(t) e^{-\lambda t}=e^{-\lambda t}\left[\frac{d}{d t}\left(W(t)(A-\lambda)^{-1}\right)-\lambda W(t)(A-\lambda)^{-1}\right],
$$

so that

$$
\begin{aligned}
W(t) & =\frac{d}{d t}\left(W(t)(A-\lambda)^{-1}\right)+(A-\lambda-A)(A-\lambda)^{-1} W(t) \\
& =\frac{d}{d t}\left(W(t)(A-\lambda)^{-1}\right)+W(t)-A(A-\lambda)^{-1} W(t) .
\end{aligned}
$$

Cancelling out $W(t)$ on both sides and integrating gives us

$$
\begin{aligned}
(W(t)-W(0))(A-\lambda)^{-1} & =A(A-\lambda)^{-1} \int_{0}^{t} W(s) d s \\
& =\int_{0}^{t} W(s) d s+\lambda(A-\lambda)^{-1} \int_{0}^{t} W(s) d s,
\end{aligned}
$$

so that $\operatorname{Im}\left(\int_{0}^{t} W(s) d s\right) \subseteq \mathcal{D}(A)$ and, applying $(A-\lambda)$ to both sides,

$$
(A-\lambda) \int_{0}^{t} W(s) d s=W(t)-W(0)-\lambda \int_{0}^{t} W(s) d s,
$$

giving us (4) of Definition 1.3.

It remains to show that there exists $g$, satisfying $(*)$, such that $g(A)$ is nontrivial.

By hypothesis, there exists a nontrivial $k \in E_{\Omega}$. Define

$$
h(z) \equiv \frac{k(z)}{(\lambda-z)^{m}} .
$$

If $h(A)$ is nontrivial, let $g \equiv h$. If $h(A)$ is trivial, then note that, since $h$ is nontrivial, there exists a connected component of $\Omega$, call it $\Omega_{0}$, such that $h(z) \neq 0$ for some $z \in \Omega_{0}$. By Proposition 3.2, $h \equiv 0$ on $\sigma(A)$. Thus $\sigma(A) \cap \Omega_{0}$ cannot have a cluster point. This implies that there exists an isolated point $\lambda_{0}$ in $\sigma(A) \cap \Omega_{0}$. Since $h$ is nontrivial on $\Omega_{0}$, the order of $\lambda_{0}$ as a zero of $h$ is finite; that is, there exists a natural number $n$ such that

$$
g(z) \equiv \frac{h(z)}{\left(\lambda_{0}-z\right)^{n}}\left(z \in \Omega, z \neq \lambda_{0}\right), \quad g\left(\lambda_{0}\right) \equiv \frac{1}{n !} h^{(n)}\left(\lambda_{0}\right)
$$

is analytic in $\Omega$ and $g\left(\lambda_{0}\right) \neq 0$. Clearly $g$ satisfies $\left(^{*}\right)$. Again by Proposition 3.2, $g(A)$ cannot be trivial. Thus $g$ is the desired function. 
Proof of Theorem 2.7. (a) $\rightarrow$ (c) is the same as the proof of $[2$, Theorem 23.6 (a) $\rightarrow$ (c)]. If $O$ is an $\alpha$-interior subset of $\Omega$, then $(A f)(z) \equiv z f(z)$, with maximal domain, on $X \equiv H^{\infty}(O)$, is an operator of $\alpha$-type $\Omega$. If $u$ is a guaranteed non-trivial solution of $(\mathrm{ACP})$, then

$$
[u(t)](z)=e^{t z}[u(0)](z)
$$

thus $u(0)$ is a nontrivial member of $E_{O}$.

(b) $\rightarrow$ (a) is clear, since $u(t) \equiv W(t) y$ is a solution of (ACP), with initial data $x=C y$, for any $y \in \mathcal{D}(A)$.

For $(\mathrm{c}) \rightarrow(\mathrm{b})$, note first that by [2, Lemma 23.12], there exists an $\alpha$-interior subset $O$ of $\Omega$ such that $A$ is of $\alpha$-type $O$. (b) now follows from Theorem 2.3.

\section{REFERENCES}

[1] E. B. Davies, "One-parameter Semigroups," London Math. Soc. Monographs 15, Academic Press, 1980. MR 82i: 47060

[2] R. deLaubenfels "Existence Families, Functional Calculi and Evolution Equations," Lecture Notes in Mathematics 1570, Springer Verlag, Berlin 1994. MR 96b:47047

[3] R. deLaubenfels, G. Sun and S. Wang, Regularized semigroups, existence families and the abstract Cauchy problem, J. Diff. and Int. Eqns. 8 (1995), 1477-1496. MR 96j:47035

[4] R. deLaubenfels, Automatic extensions of functional calculi, Studia Math. 114 (1995), 237259. MR 96f: 47029

[5] J. A. Goldstein, "Semigroups of Linear Operators and Applications," Oxford, New York, 1985. MR 87c: 47056

[6] A. Pazy, "Semigroups of Linear Operators and Applications to Partial Differential Equations," Springer, New York, 1983. MR 85g:47061

[7] J. A. van Casteren, "Generators of Strongly Continuous Semigroups," Research Notes in Mathematics 115, Pitman, Boston, 1985. Zbl. 576:47023

Scientia Research Institute, P. O. Box 988, Athens, Ohio 45701

E-mail address: 72260.2403@compuserve.com

Department of Mathematics, Nanjing University, Nanjing, Jiangsu 210008, People's Republic OF China

E-mail address: wang2598@netra.nju.edu.cn 